\title{
Putting ‘Local’ Back into Public Wifi Hotspots
}

\author{
Matthias Korn and Clemens Nylandsted Klokmose \\ Department of Computer Science, Aarhus University \\ Aabogade 34, 8200 Aarhus N, Denmark \\ \{mkorn, clemens\}@cs.au.dk
}

\begin{abstract}
Public Wifi hotspots in cafes and public places are based on wireless local area network technology (WLAN). In contrast to the common understanding of connecting directly to the internet when connecting to a Wifi hotspot, we are proposing to bring the original notion of connecting to a local network back to the fore. By hooking into the act of a user connecting to a local network, we see a design space emerge that allows us to spur various (off- and on-line) activities in a hyperlocal context. We propose to (re-)explore this notion of the locality of networks in the age of the ubiquitous Wifi hotspot in cafes, bars, community centers, and other (semi-)public places in order to facilitate co-located activities for such varied purposes as fostering local community, civic participation, sociality in general, and entertainment. We propose a network locality that builds on local infrastructure (WLAN) and combines personal devices (mobile phones) with stationary interactive surface technology (wall displays and tables) to facilitate social on- and off-line interactions in local settings. In this position paper, we outline technological opportunities associated with this idea as well as envisioned usage scenarios in different settings.
\end{abstract}

\section{Author Keywords}

Wireless local area networks, network locality, co-located sociality, participation.

\section{ACM Classification Keywords}

H.5.2 Information interfaces and presentation (e.g., HCI): Miscellaneous.

\section{INTRODUCTION}

Wireless local area network technology (WLAN) is widely and openly deployed on Wifi hotspots in many private and public places (from malls and cafes to citizen service centers and public squares). Largely, the sole purpose of these Wifi hotspots for its users today is to access the internet. However, when users connect to such hotspots, they do not connect to the internet directly, but first and foremost to a local network that happens to provide internet access as well. We seek to exploit this notion of a (re-)localized network (cf. [3]) in future designs of technology for participation and engagement on local topics. We believe a wireless network

Copyright is held by the author/owner(s). UbiComp '12, September 5-8, 2012, Pittsburgh, USA. ACM 978-1-4503-1224-0/12/09. could provide the anchor point for co-located computer-mediated participation, where physically and digitally mediated interaction can be bridged.

Therefore, we imagine that the act of connecting to the wireless network could be used to lure people into participation on relevant local topics. We already see ad-supported free Wifi where access is granted, e.g., only after watching a 30second commercial. In a non-commercial context this could rather be used for encouraging social on- and off-line interactions and civic participation with a strong emphasis on the local context. A central aspect is, however, to make the local network reach beyond the online interactions and make it graspable in the physical space through physical installations (e.g. interactive wall and table surfaces). Such physical representations do not only provide 'access' to the activities for those without Wifi-enabled devices, but may in general attract people to engage in more direct offline interactions. This aspect of physical representations and social offline interactions is important if we seek to emphasize the hyperlocal context where participation should take place.

A large body of work has investigated the effects of Wifi networks in public and semi-public spaces (e.g. [2, 5, 6]). While drawing inspiration from this previous work, we suggest to rather understand the notion of localized networks as a new design space. A domain where such possibilities have already been explored is local music sharing $[1,4,7]$.

\section{TECHNOLOGICAL BASIS}

Many people connect to open Wifi networks automatically or semi-automatically through their laptops or smart phones. The technology already offers the possibility to send people to a proxy page once they open a browser after having connected to the WLAN. This anchor can be further exploited through modern web technology. While past ubicomp systems have heavily relied on custom software for participation, modern web-technology allows for more and more interactive applications directly in the browser. Even peer-topeer network technology, which is one of the core technologies in ubicomp, will be integrated in our browsers in the near future ${ }^{1}$. This means that people will be able to directly interact through their mobile devices, without having to install any custom software.

We believe the key to augmenting co-located participation

\footnotetext{
${ }^{1}$ http://www.w3.org/2011/04/webrtc-charter. html
} 
through technology is to bridge interaction on personal devices, i.e., those that people use to connect to the network (e.g. laptops or smart phones), with public devices such as shared interactive wall or table surfaces. Personal devices may hereby provide an individual perspective into the local activity (e.g. others' comments to own postings and participating debates), while shared surfaces may provide a collective perspective (e.g. trending articles, hot debates, recent comments) and incite further co-located offline interactions. The challenge lies in creating a combined experience between shared interactive surfaces and personal devices.

\section{EXAMPLE USAGE SCENARIOS}

We envision a number of scenarios where a focus on local context in the use of Wifi hotspot could create a difference.

\section{Local participation at the regional community centre}

We envision to use our idea to provide citizens in a new and still forming national park with a shared forum for exchange that has close ties to the area. By attaching a distinctively local component to an existing Wifi network in a frequented public or semi-public place such as a community/visitor centre, a cultural institution, or a popular cafe, we seek to engage users of the network in the development of the park through various means for civic participation (e.g. discussions, story telling, voting, etc.). Upon joining the network, people are automatically directed to a local web-based participation platform. Interactive wall and/or table surfaces in the locality make participation visible in the physical space and accessible beyond personal devices carried by citizens in order to encourage ad-hoc offline interactions. The wall display shows people participating through their personal devices live in order to spur offline discussions taking a vantage point in their comments. Due to the geographical spread of the park, we envision a possibility where networks at different locations are joined to span a larger common network forming a shared forum for exchange throughout the park.

\section{Student politics at the student bar on campus}

Student politics could be discussed at the student bar on campus. Upon joining the wireless network, a local annotation and discussion layer could be added on top of the web allowing students to comment and discuss the media they consume through the web with their peers in the bar. $\mathrm{Na}$ tional newspapers and other online media reporting about the university, student laws, and other topics of primary interest to students could be the focus. A portal could give an overview of the articles and websites discussed in the bar and articles could be curated by the bar to spur debates of relevance to the university. The university itself could further use the system to increase codetermination by students on various university levels. Active students could choose to have their avatars represent the table they currently sit at on the wall surface in order to encourage offline interactions. Hot topics or burning debates could be visualized on the wall surface as well. Lastly, the wall display could show concrete possibilities for offline interactions here and now, e.g., by creating meeting points and matching people with similar interests; or listing contact persons and open consultation hours nearby.

\section{Entertainment at a bar}

Similarly, the system could just as well be used for entertainment: doing bar quizzes, games, and other forms of interactive activities where people could participate through their mobile devices just as well as through installed interactive wall surfaces or surfaces integrated into bar tables. Points for games and quizzes could be associated with avatars of visitors and their table visualized on the wall.

\section{SUMMARY}

In this paper, the concept of (re-)localized networks in the form of the ubiquitous Wifi hotspot is brought forward and exemplified through various settings and purposes. We propose to shift focus in the design of Wifi hotspots from merely providing internet access independent of (and leading away from) the physical locality towards providing a platform for participation encouraging social on- and offline interactions within the hyperlocal context of the locality. In the light of civic participation and community building this could potentially provide new ways of engagement on the local level.

A number of challenges are yet to be addressed: How does one move from the largely solitary use of personal devices to social offline interactions at places where people are often anonymous? How can a feeling of togetherness be transferred to offline interactions, when it is largely defined in (and mediated through) the online world? We plan to further explore these ideas and challenged within the new centre on Participatory Information Technology at Aarhus University: http://pit.au.dk/.

\section{REFERENCES}

1. Bassoli, A., Moore, J., and Agamanolis, S. tunA: Synchronised music-sharing on handheld devices. In Adjunct Proc. Ubicomp 2004 (2004).

2. Forlano, L. When Code Meets Place: Collaboration and Innovation at WiFi Hotspots. PhD thesis, Columbia University, 2008.

3. Gordon, E., and de Souza e Silva, A. Net Locality: Why Location Matters in a Networked World. Wiley-Blackwell, Boston, MA, USA, 2011.

4. Håkansson, M., Rost, M., and Holmquist, L. E. Gifts from friends and strangers: A study of mobile music sharing. In Proc. ECSCW 2007, Springer (2007), 311-330.

5. Hampton, K. N., and Gupta, N. Community and social interaction in the wireless city: wi-fi use in public and semi-public spaces. New Media \& Society 10, 6 (2008), 831-850.

6. Hampton, K. N., Livio, O., and Goulet, L. S. The social life of wireless urban spaces: Internet use, social networks, and the public realm. Journal of Communication 60, 4 (2010), 701-722.

7. O’Hara, K., Lipson, M., Jansen, M., Unger, A., Jeffries, H., and Macer, P. Jukola: democratic music choice in a public space. In Proc. DIS 2004, ACM Press (2004), 145-154. 